\title{
Una metodología, con niveles de jerarquía, propuesta para el diseño de la distribución en plantas industriales
}

\author{
G.A. Pérez ${ }^{1 *}$, J.R. Medina ${ }^{2}$ y R.L. Romero ${ }^{3}$ \\ ${ }^{1}$ Facultad de Ingeniería Química (UNL) e INGAR (CONICET-UTN) \\ Santiago del Estero 2829-(3000) Santa Fe, Argentina \\ E-mail: gperez@unl.edu.ar \\ ${ }^{2}$ Facultad de Ingeniería Química, Universidad Nacional del Litoral (UNL) \\ Santiago del Estero 2829-(3000) Santa Fe, Argentina \\ E-mail: jrmedina@fiqus.unl.edu.ar \\ ${ }^{3}$ Facultad de Ingeniería Química (UNL) e INTEC (CONICET-UNL) \\ Santiago del Estero 2829-(3000) Santa Fe, Argentina \\ E-mail: tatin@santafe-conicet.gov.ar
}

(recibido/received: 30-Agosto-2007; aceptado/accepted: 23-Abril-2008)

\section{RESUMEN}

El ingeniero que participa en la definición de la distribución en una planta piensa principalmente en aspectos de seguridad y económicos. En este trabajo se desarrolla un marco conceptual para descomponer y resolver los pasos de la distribución. El primer paso en un primer nivel de descomposición, se relaciona con los códigos, leyes y normas que regulan la construcción, porque son obligatorias en aspectos básicos y requerimientos de medio ambiente, seguridad y salud de los operarios. Seguidamente se refiere al tipo de construcción industrial, que define el conjunto. El segundo nivel, plantea el diseño de la planta de procesos, dónde las consideraciones de diseño actúan como restricciones a la función objetivo, representada por el costo total anual de operación, que debe minimizarse. Subsecuentemente, se vuelve a iterar para minimizar las distancias entre los sectores de planta involucrados. El procedimiento resultó adecuado para todo tipo de plantas industriales.

Palabras claves: analísis en dos niveles; distribución; optimización de costos; planta de procesos

\begin{abstract}
When a process engineer is involved in a plant layout definition, he or she always thinks in a safe and economic design. In this work, a conceptual framework to split up and solve a two levels layout design methodology was developed. The initial step at the first level is related to codes or rules that constraint the design and construction, because they are mandatory in some basic aspects, as the environmental, health and safety requirements. The next step, in this first level, is the decision about the type and characteristics of the industrial building. The second level is the battery limits layout, where the design considerations act as constraints and the target is the annual cost minimisation. Subsequently, an iteration that involves a distance minimisation between the different sectors must be accomplished. The procedure was appropriate for any industrial plants.
\end{abstract}

Keywords: analysis in two levels; battery limits; cost optimisation; layout

*Autor para la correspondencia 


\section{INTRODUCCIÓN}

Durante la ejecución de la Ingeniería Básica, aparece el diseño de la planta de procesos como un hecho relevante de esta fase de la evolución del proyecto. La economía alcanzada y el futuro desempeño de la instalación son el resultado del estado actual en la materia y de las decisiones tomadas por el ingeniero de procesos en esta etapa; lo que indica que hay un continuo aprendizaje en este sentido. Es un típico problema con múltiples objetivos y no hay un algoritmo adecuado para resolver problemas prácticos concretos (Yang y Kuo, 2003; Lewis y Marron, 1974).

La mayoría de los trabajos clásicos publicados están basados en la experiencia práctica (ver, por ejemplo, Mecklenburgh, 1985). Pero, en los últimos años, el problema ha sido tratado a partir de modelación matemática y como un caso interesante de optimización, considerando diferentes aspectos (Barbosa-Póvoa et al., 2001; Georgiadis y Macchietto, 1997; Georgiadis et al., 1999; Patsiatzis y Papageorgiou, 2003; Realff et al., 1996), no siendo ninguno de ellos representativo de la problemática que se presenta al ingeniero de procesos al encarar una distribución (layout) de planta, ni contemplar la multidisciplina intrínseca que tiene el problema.

Por otro lado, una metodología es más conveniente porque puede incorporar metas cualitativas y cuantitativas en el proceso de diseño, lo que da lugar a la consideración de regulaciones o normas taxativas que se aplican a este caso. Por supuesto, que el éxito del resultado bajo esta mecánica dependerá de la capacidad y la experiencia del ingeniero a cargo, razón por la cual este trabajo trata de clarificar el punto de vista y el conocimiento necesario que emplea el ingeniero de proceso en estos diseños. Es secuencial por naturaleza debido a las restricciones tan diversas que imponen las consideraciones de seguridad, por un lado y las económicas por otro.

Nadie puede reducir los costos anuales sin antes dar cumplimiento a la legislación vigente en la construcción de plantas, o a consideraciones relacionadas con el riesgo eléctrico inherente a cada sector de planta, en cada caso específico, por ejemplo, como las normas NEC (Código Eléctrico de los Estados Unidos, por sus siglas en inglés) o las de OSHA (Agencia Estadounidense de Seguridad y Salud Ocupacional, por sus siglas en inglés) (Leblanc y Lawrence, 2000) que clasifica las áreas de acuerdo a las sustancias químicas que intervienen en el proceso. Las consideraciones económicas, de suma importancia, son las referidas a aspectos como: mantenimiento, operatividad, construcción, control, expansiones futuras, etc.

\section{DISCUSIÓN DE LA PROPUESTA}

En un primer nivel de análisis se deben definir todos los sectores de la industria, esto es, las plantas auxiliares, los depósitos, plantas de tratamiento, etc.

En un segundo nivel, el diseño de la planta en sí (battery limits) es la meta más interesante desde un punto de vista económico. En este paso es necesario contar con las hojas de especificaciones de todos los equipos de procesos, los diagramas $\mathrm{P} \& \mathrm{I}$ (tuberías e instrumentos), y los planos isométricos de las tuberías o los gráficos CAD (diseño asistido por computadora) elaborados a tal fin.

El criterio es obtener una planta de procesos con un costo total anual mínimo (Patsiatzis y Papageorgiou, 2002), y que sea compatible con las condiciones de seguridad, confiabilidad y operatividad.

La aplicación de las restricciones, en algún orden determinado, no es la práctica usual. Se analizan alrededor de cada equipo o de un área particular (Baasel, 1976); y los criterios están dados por:

1. Mantenimiento

2. Operatividad

3. Futuras expansiones

4. Seguridad y confiabilidad

5. Requerimientos de la construcción y el montaje

6. Tuberías y flujos de materiales 
Consecuentemente, este procedimiento sugiere la aplicación de los mismos siguiendo un orden de prioridad, para lograr la verificación que los convalide. Dicha prioridad se impondrá de acuerdo a la industria considerada. Esto es:

Plantas de productos básicos o pseudo-básicos químicos y/o petroquímicos

Una vez concluido el primer nivel de análisis, basado en la clasificación de áreas y medidas de seguridad, se centra el trabajo en las conexiones, esencialmente tuberías, entre equipos, teniendo presente un diagrama de flujos dado con una distribución del tipo lineal, en $\mathrm{L}$, etc. Con la consideración de los tubos, tanto de procesos como auxiliares, se define el armazón para las tuberías (piperack) y se prevén los espacios necesarios, entre equipos, para construcción y mantenimiento, preferentemente en este orden. La operatividad no se ve restringida, en general, en estas plantas por la forma en que son controladas y operadas.

\section{Industrias alimenticias}

Se atiende primero a las reglas que imponen la FDA (Agencia Estadounidense para la Administración de Alimentos y Fármacos, por sus siglas en inglés), o el Codex Alimentarius correspondiente. Una vez cumplido este requisito se enfatiza en las condiciones de asepsia y en el mantenimiento (que incluye el sistema CIP: "cleaning-in-place"); para definir la distribución de tuberías y accesorios de acuerdo a la manera más económica dentro de los edificios.

\section{Industrias de la química fina y farmacéuticas}

En el segundo nivel el énfasis es puesto en operatividad (flexibilidad y manipulación de materiales), habida cuenta de la condición multiproducto $\mathrm{y} / \mathrm{o}$ multi-proceso que son $\mathrm{y}$ cobra importancia el arreglo de los equipos. Las condiciones de seguridad quedan definidas en un primer nivel cuando se definen las zonas, el tipo de construcción, acorde con las normativas de la ANMAT (Administración Nacional de Medicamentos, Alimentos y Tecnología Médica de Argentina), FDA, etc.
De lo manifestado se desprende que se ha impuesto el mencionado orden de jerarquías para la aplicación de los criterios, especificando el tipo de industria que se trata. Este procedimiento se complementa, en los casos más complejos, con técnicas de comparación entre pares, como la difundida metodología del Proceso de Jerarquía Analítica (Hafeez et al., 2002), simplificando de este modo al problema multi-variable y evitando una resolución matemática compleja, que no puede garantizarse sea eficiente y precisa (Fuh-Hwa y Hui, 2005).

Como se mencionó, el procedimiento es secuencial, basado en las definiciones preliminares y en las restricciones que imponen las distintas regulaciones, en cada caso. En el momento de hacer el trabajo se cuenta con la información desarrollada durante la Ingeniería Básica, que comprende:

1. Necesidad de instalaciones y servicios auxiliares

2. Edificios

3. Tratamiento de efluentes

4. Áreas de servicio y de almacenamiento

5. Consideraciones de la planta de procesos

6. Características del sitio y del suelo

7. Condiciones del clima

8. Vientos predominantes y dominantes

Con todos estos datos es posible formular las definiciones de la implantación general (plot plan) preliminar. El paso siguiente es la definición y el diseño de la planta propiamente dicha (battery limits). En este caso, el espacio total considerado en el análisis es el correspondiente al sector de proceso o de manufactura.

Los otros sectores han sido estimados, de modo que el dimensionamiento preliminar (tamaño global) de las diferentes áreas se ha cumplido. Al tener la primera propuesta de trabajo se tiene en cuenta el análisis de las distancias entre los diferentes sectores de toda la planta.

Ahora entran en juego los criterios relacionados con expansiones futuras, caminos internos de diferentes propósitos, etc.; y la posición relativa de los diferentes sectores de acuerdo a la 
funcionalidad, la relación con el proceso, los efluentes, los vientos predominantes, etc. Finalmente, una minimización de las distancias se lleva a cabo para satisfacer el objetivo de costos. Se ve más claramente el procedimiento en la Fig. 1, en donde se ilustra la metodología y la interrelación de las decisiones.

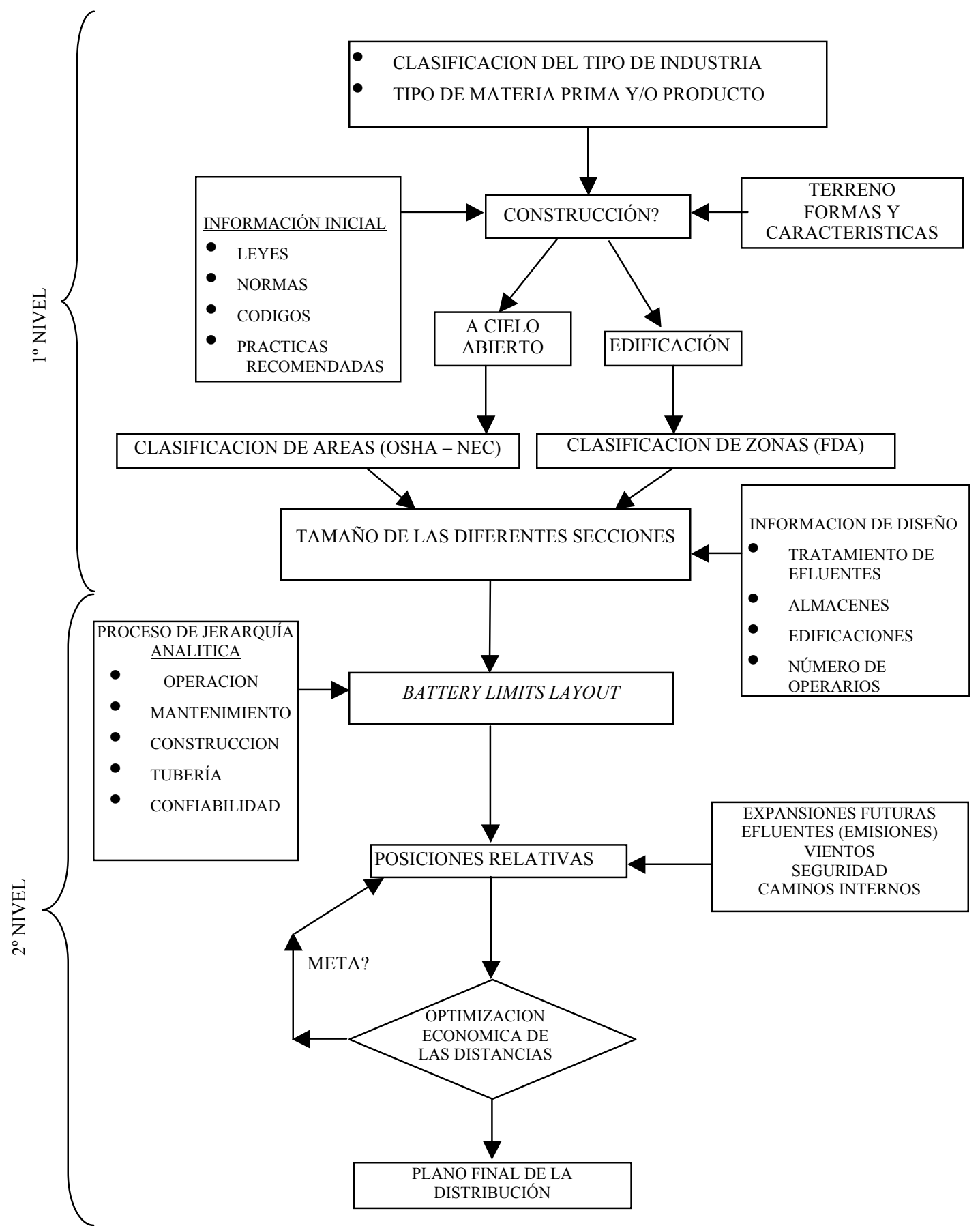

Fig. 1 Diseño del procedimiento. 


\section{ANÁLISIS DE LA METODOLOGÍA}

Las definiciones preliminares son las más importantes porque deben tener en cuenta las regulaciones y toda la información inicial. Este procedimiento asume que la seguridad, en todo sentido, es el primer nivel $\mathrm{y}$ todas las consideraciones acerca del diseño de la implantación (plot plant) se relacionan directamente con esta cuestión. La elección del tipo de edificio o construcción, los materiales, los pisos, el acabado de paredes y techos, cuando los hubiera, es la decisión que sigue, y las restricciones son las imposiciones de las diferentes normas específicas en cada caso.

El siguiente paso, que es el más importante desde el punto de vista del diseño, define los tamaños de las diferentes zonas de la planta, según las hojas de especificaciones y las características de los servicios auxiliares, como caldera, torre de enfriamiento, tratamiento de efluentes, antorcha, etc. El conocimiento de la estructura de la organización es, también, necesario en este paso para resolver las comodidades requeridas en la obra civil que resulte.

Luego, la tarea más compleja es el diseño de la distribución de equipos en el área de procesos. Se detallan todas las consideraciones concernientes a aspectos económicos, de una manera directa o indirecta.

Así, cuestiones de operatividad y mantenimiento fueron evaluadas en una forma directa $y$ confiabilidad o disponibilidad son buenos ejemplos de los aspectos denominados en segundo lugar.

Por esta razón, este trabajo define un orden de prioridad en la aplicación de los criterios para resolver el diseño de la planta propiamente dicha. De este modo, se consideran los parámetros relevantes en cada caso específico, principalmente desde el punto de vista económico.

Algunas de las metas posibles a fijar, para aplicar el procedimiento son:
1. Minimizar el tiempo total de producción

2. Proveer a los empleados comodidad y seguridad operacional

3. Minimizar los costos de traslado de materiales

4. Toda otra que cada situación amerite

La ubicación, la distancia entre equipos y su posición relativa, sujetas a las restricciones fijadas son el resultado final del diseño.

Con la definición total de los tamaños de las áreas, incluyendo la planta del procesos, el procedimiento sigue con la determinación de la posición relativa entre ellas. En necesario tener en cuenta la circulación y las conexiones existentes entre ellas; siendo la distancia y el costo asociado a esta la variable de diseño a manipular.

Una apropiada minimización de distancias $d\left(X, P_{i}\right)$ (Francis y White, 1974), es la dada por la configuración rectilínea, porque los caminos ocurren a lo largo de islas conectadas según un patrón rectangular.

La ecuación representativa, donde $\mathrm{X}(x, y)$ es la variable con sus coordenadas cartesianas y $\mathrm{P}$ $\left(a_{i}, b_{i}\right)$ es el punto fijo elegido con sus coordenadas, resultando:

$$
d\left(X, P_{i}\right)=\left|x-a_{i}\right|+\left|y-b_{i}\right|
$$

Si ahora se sigue con la inclusión de los costos (w), asociados a las distancias, el conjunto de configuraciones a minimizar resulta:

$$
\begin{aligned}
& \min f_{1(x)}=\sum_{i=1}^{m} w_{i}\left|x-a_{i}\right| \\
& \min f_{2(y)}=\sum_{i=1}^{m} w_{i}\left|y-b_{i}\right|
\end{aligned}
$$

donde las funciones $\left(\mathrm{f}_{\mathrm{i}}\right)$ evaluadas son objetivos económicos. Una vez que las funciones objetivos $\left(\mathrm{f}_{\mathrm{i}}\right)$ se satisfacen al hallarse el mínimo, el procedimiento termina y se obtiene una versión preliminar de la distribución (layout). El último paso es el análisis detallado en una 
versión CAD, de esta así obtenida, con el propósito de verificar todas las hipótesis, su cumplimiento y la consistencia de los resultados.

\section{CONCLUSIONES}

El presente trabajo está destinado a discutir una metodología práctica para desarrollar un diseño completo de una distribución (layout). Tomando las definiciones preliminares, y basándose en el "estado actual" y el sentido común de un diseñador experto, se establece una estrategia, que resulta, de una manera explícita, en una estructura secuencial de diseño.

El procedimiento es una relación de compromiso entre los aspectos económicos y de seguridad que definen una implantación y enfatiza las consideraciones de más relieve que aparecen en el diseño de la planta de procesos propiamente dicha. Para ello, se establece una forma jerárquica de la aplicación de los diferentes criterios de diseño, según el caso tratado.

Finalmente, una técnica usual de minimización de costos, mediante la optimización de las distancias, completa la implantación general del complejo.

\section{AGRADECIMIENTOS}

Los autores agradecen a la Universidad Nacional del Litoral (UNL) y al Consejo Nacional de Investigaciones Científicas y Técnicas (CONICET), por su apoyo para producir este trabajo.

\section{REFERENCIAS}

Baasel, W.D. (1976). Preliminary Chemical Engineering Plant Design. Elsevier North Holland, Inc. New York, USA.

Barbosa-Póvoa, A.P., R. Mateus y A.Q. Novais (2001). Optimal Two-dimensional Layout of Industrial Facilities. International Journal of Production Research, Vol. 39, No. 12, pp. 25672593.
Francis, R.L. y J.A. White (1974). Facility Layout and Location: An Analytical Approach. Prentice-Hall, Inc., Englewood Cliffs. New Jersey, USA.

Fuh-Hwa, F.L. y L.H. Hui (2005). The Voting Analytic Hierarchy Process Method for Selecting Supplier. International Journal of Production Economics, Vol. 97, No. 03, pp. 308317.

Georgiadis, M.C. y S. Macchietto (1997). Layout of Process Plants: A Novel Approach. Computers and Chemical Engineering, Vol. 21, Supplement 1, pp. 337-342.

Georgiadis, M.C., G. Schilling, G.E. Rotstein y S. Macchietto (1999). A General Mathematical Programming Approach for Process Plant Layout. Computers and Chemical Engineering, Vol. 23, No. 07, pp. 823-840.

Hafeez, K., Y. Zhang y N. Malak (2002). Determining Key Capabilities of Firm using Analytic Hierarchy Process. International Journal of Production Economics, Vol. 76, No. 01, pp. 39-51.

Leblanc, J.A. y W.G. Lawrence (2000). Benefit from the Three-zone National Electrical Code. Chemical Engineering Process, Vol. 96, No. 12, pp. $75-82$.

Lewis, B.T. y J.P. Marron (1974). Facilities and Plant Engineering Handbook. Chapter 04, pp. 257. McGraw-Hill Education. New Jersey, USA.

Mecklenburgh, J.C. (1985). Process Plant Layout. John Wiley and Sons, Inc. New York, USA.

Patsiatzis, D.I. y L.G. Papageorgiou (2002). Optimal Multi-floor Process Plant Layout. Computers and Chemical Engineering, Vol. 26, No. 04, pp. 575-583.

Patsiatzis, D.I. y L.G. Papageorgiou (2003). Efficient Solution Approaches for the Multifloor Process Plant Layout Problem. Industrial and Engineering Chemistry Research, Vol. 42, No. 04, pp. 811-824. 
Realff, M.J., N. Shah y C.C. Pantelides (1996). Simultaneous Design, Layout and Scheduling of Pipeless Batch Plants. Computers and Chemical Engineering, Vol. 20, No. 06, pp. 869-883.

Yang, T. y C. Kuo (2003). A Hierarchical AHP/DEA Methodology for the Facilities Layout Design Problem. European Journal of Operational Research, Vol. 147, No. 01, pp. 128-136.

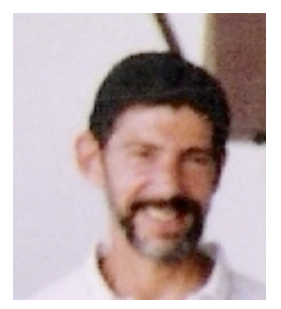

Gustavo A. Pérez es Ingeniero Químico, recibido en la Universidad Nacional del Litoral, UNL (Argentina). Desarrolla sus actividades como Profesor Titular de las asignaturas ingeniería económica y proyecto industrial en la Facultad de Ingeniería Química. Además realiza tareas de investigación en el CONICET, en el área de ingeniería y economía de procesos.

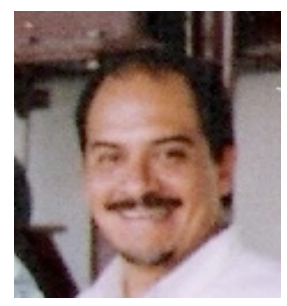

José R. Medina es Ingeniero Químico y Magíster en Ciencia y Tecnología de Alimentos, recibido en la Universidad Nacional de Litoral (Argentina). Participa como Profesor Adjunto en las asignaturas de ingeniería económica y proyecto industrial, y con tareas de investigación en tecnologías de aprovechamiento de los recursos pesqueros de agua dulce.

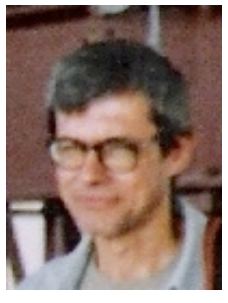

Roberto L. Romero es Ingeniero Químico y Magíster en Ingeniería Química de la Universidad Nacional del Litoral, UNL (Argentina). Desarrolla actividades docentes en las asignaturas ingeniería económica $\mathrm{y}$ proyecto industrial en dicha universidad. Realiza tareas de investigación en el CONICET, en el área de ingeniería de las foto-reacciones y los foto-reactores. 